\title{
Weak reachability and controllability of discrete-time nonlinear systems: generic approach and singular points
}

\author{
T. Mullari, U. Kotta, Z. Bartosiewicz, M.A. Sarafrazi, C.H. Moog \& E. Pawluszewicz
}

February 13, 2018

\begin{abstract}
The paper finds the singular points from which (to which) the generically accessible system is not weakly reachable (controllable) in $k$ steps. These points are found with the help of the space of vector fields, being the discrete-time analogue of the strong accessibility distribution. Unlike in the continuous-time case, separate object is needed to find the singular points related to weak reachability.
\end{abstract}

\section{KEYWORDS}

nonlinear system, weak reachability, weak controllability, discrete-time, singularities.

\section{Introduction}

The generic accessibility property of non-linear discrete-time system can be characterized using the concept of autonomous variables, see e.g. (Aranda-Bricaire, Kotta and Moog, 1996), (Mullari, Kotta, Bartosiewicz, Pawluszewicz and Moog, 2017). The existence of an autonomous variables can be easily checked with the help of the vector space of 1-forms, denoted by $\mathcal{H}_{\infty}$, and introduced in (Aranda-Bricaire, Kotta and Moog, 1996). The accessibility property is known to be related to reachability and controllabiliy properties. Unfortunately, $\mathcal{H}_{\infty}$ does not allow to find the singular points, from which (to which) the generically accessible system is not weakly reachable (controllable). In the continuous-time case alternative accessibility condition (Conte, Moog, Perdon, 2007) is formulated in terms of strong accessibility distribution, that allows to find the reachability singular points. In the discrete-time case the space of vector fields $\mathcal{D}_{\infty}^{\pi}$ (Mullari, Kotta, Bartosiewicz, Pawluszewicz and Moog, 2017) is the discrete-time analogue of the strong accessibility distribution. Computing of $\mathcal{D}_{\infty}^{\pi}$ requires to define the backward shift operators acting on the vector fields in a manner that is consistent with the geometric meaning of shifting the vector field along the (discrete) trajectory of the control system (Rieger, Schlacher, 2011). However, unlike the continuous-time case, $\mathcal{D}_{\infty}^{\pi}$ cannot be directly used for finding the singular points from which the system is not weakly reachable in $k$ steps. The present paper builds upon the results of (Mullari, Kotta, Bartosiewicz, Pawluszewicz and Moog, 2017), in particular on $\mathcal{D}_{\infty}^{\pi}$. This vector space is the starting point to construct the matrix which helps to find the reachability singular points. Expressing $\mathcal{D}_{\infty}^{\pi}$ in a matrix form and shifting its elements as functions forward a necessary number of steps allows to find the singular points $x$ from which the system is not weakly reachable.

Unfortunately, this matrix does not enable to find the autonomous variables. So, in 
the discrete-time case, unlike the continuous-time case, one needs two objects. The first one to check generic accessibility and to find the autonomous variables, and the second one to check generic accessibility and to find the reachability singular points. Moreover, if a state transition map of a system has a global analytic inverse with respect to $x$, the first object, the vector space $\mathcal{D}_{\infty}^{\pi}$ enables also to determine the controllability singular points, to which the generically accessible system is not weakly controllable.

Finally, in (Hanba, 2017) it has been proven that controllability to the origin is equivalent to state feedback stabilizability. Necessity of controllability to the origin can, for instance, be checked using the results of this paper. As for the other results on reachability and controllability, see (Kawano, Ohtsuka, 2016), (Kawano, Ohtsuka, 2013). These papers developed conditions for reachability (controllability) from an equilibrium point (to an equilibrium point) for the subclass of polynomial discrete-time systems.

\section{Preliminaries}

Consider the discrete-time nonlinear control system

$$
x^{\langle 1\rangle}(t)=\bar{\Phi}(x(t), u(t))
$$

where $x^{\langle 1\rangle}(t):=x(t+1), t \in \mathbb{Z}$, the variables $x(t) \in \bar{X} \subset \mathbb{R}^{n}, u(t) \in U \subset \mathbb{R}^{m}$ and the state transition map $\bar{\Phi}: \bar{X} \times U \rightarrow \bar{X}$ is supposed to be analytic. Both $\bar{X}$ and $U$ are assumed to be open in respective sets. Recall below some facts from (Mullari, Kotta, Bartosiewicz, Pawluszewicz and Moog, 2017). Denote by $\mathcal{K}$ the set of meromorphic functions in a finite number of independent variables from the infinite set $\mathcal{C}=\left\{x, u^{\langle k\rangle}, z^{\langle-l\rangle}, k \geq 0, l>0\right\}$. Here $u^{\langle k\rangle}$ denotes the $k$-th order forward shift of $u, z^{\langle-l\rangle}$ the $l$-th order backward shift of a variable $z$, defined below.

Assumption 1. The map $\bar{\Phi}$ can be extended to $\Phi=\left(\bar{\Phi}^{T}, \chi^{T}\right)^{T}: \bar{X} \times U \rightarrow \bar{X} \times \mathbb{R}^{m}$ so that $\Phi$ has a global analytic inverse $\Phi^{-1}$, defined on its image $\Phi(\bar{X} \times U)$.

In some cases the existence of a local inverse of $\Phi$ could suffice and this could be guaranteed by some rank condition. However, the field $\mathcal{K}$ consists of global functions, so to define forward and backward shifts of $\mathcal{K}$ we need that $\Phi$ be globally invertible. To conclude, Assumption 1 is a bit more restrictive than the submersivity assumption, but less restrictive than the assumption of invertibility of the state transition map $\bar{\Phi}$, i.e. Assumption 2 in Section 5. The extended system has a form

$$
x^{\langle 1\rangle}(t)=\bar{\Phi}(x(t), u(t)), \quad z(t)=\chi(x(t), u(t)) ;
$$

see (Mullari, Kotta, Bartosiewicz, Pawluszewicz and Moog, 2017) for more information. Due to Assumption 1 one can define from (2) the backward shifts of $x$ and $u$ :

$$
x^{\langle-1\rangle}=\Psi\left(x, z^{\langle-1\rangle}\right), \quad u^{\langle-1\rangle}=\lambda\left(x, z^{\langle-1\rangle}\right),
$$

where $\Psi$ and $\lambda$ are the first and the second vector components of the map $\Phi^{-1}$, respectively. The forward and backward shift operators $\sigma_{\Phi}: \mathcal{K} \rightarrow \mathcal{K}$ and $\rho_{\Phi}: \mathcal{K} \rightarrow \mathcal{K}$, respectively, are defined as follows. The forward shift of a function $\varphi\left(x, u, \ldots, u^{\langle k\rangle}, z^{\langle-1\rangle}, z^{\langle-2\rangle}, \ldots, z^{\langle-l\rangle}\right) \in \mathcal{K}$ is, according 
to $(2)$, the composition $\varphi^{\langle 1\rangle}:=\varphi^{\sigma_{\Phi}}=\varphi\left(\bar{\Phi}(x, u), u^{\langle 1\rangle}, \ldots, u^{\langle k+1\rangle}, \chi(x, u)\right.$, $\left.z^{\langle-1\rangle}, \ldots, z^{\langle-l+1\rangle}\right)$, and the backward shift of a function $\bar{\varphi}\left(x, u, u^{\langle 1\rangle}, \ldots, u^{\langle k\rangle}, z^{\langle-1\rangle}\right.$, $\left.z^{\langle-2\rangle}, \ldots, z^{\langle-l\rangle}\right) \in \mathcal{K}$ according to $(3)$ the composition $\bar{\varphi}^{\langle-1\rangle}:=\bar{\varphi}^{\rho_{\Phi}}=$ $=\quad \bar{\varphi}\left(\Psi\left(x, z^{\langle-1\rangle}\right), \lambda\left(x, z^{\langle-1\rangle}\right), u, \ldots, u^{\langle k-1\rangle}, z^{\langle-2\rangle}, \ldots, z^{\langle-l-1\rangle}\right) . \quad$ Obviously,

$\sigma_{\Phi} \circ \rho_{\Phi}=\rho_{\Phi} \circ \sigma_{\Phi}=\mathrm{id}$. Define the space $\mathcal{E}^{*}$, whose elements are the vector fields of the form

$$
\Xi=\sum_{i=1}^{n} \alpha_{i} \frac{\partial}{\partial x_{i}}+\sum_{k \geq 0} \sum_{j=1}^{m} \beta_{j k} \frac{\partial}{\partial u_{j}^{\langle k\rangle}}+\sum_{l>0} \sum_{q=1}^{m} \gamma_{q l} \frac{\partial}{\partial z_{q}^{\langle-l\rangle}} .
$$

Denote by $\mathbf{X}$ the subspace of $\mathcal{E}^{*}$ spanned over $\mathcal{K}$ by $\partial / \partial x_{i}$, by $\mathbf{U}$ the subspace consisting of the vector fields $\Xi$ for which $\alpha_{i}=0$ and $\gamma_{q l}=0$, and by $\mathbf{Z}$ the subspace consisting of the vector fields $\Xi$ for which $\alpha_{i}=0$ and $\beta_{j k}=0$. Then $\mathcal{E}^{*}=\mathbf{X} \oplus \mathbf{U} \oplus \mathbf{Z}$. Define also $\mathbf{U}_{0}:=\operatorname{span}_{\mathcal{K}}\{\partial / \partial u\}$ and $\mathbf{Z}_{0}:=\operatorname{span}_{\mathcal{K}}\left\{\partial / \partial z^{\langle-1\rangle}\right\}$.

The concept of backward shift has been extended to the vector fields in (Mullari, Kotta, Bartosiewicz, Pawluszewicz and Moog, 2017). The backward shift of a vector field (4) is

$$
\Xi^{\langle-1\rangle}=\sum_{i=1}^{n} a_{i} \frac{\partial}{\partial x_{i}}+\sum_{k \geq 0} \sum_{j=1}^{m} b_{j k} \frac{\partial}{\partial u_{j}^{\langle k\rangle}}+\sum_{l>0} \sum_{q=1}^{m} c_{q l} \frac{\partial}{\partial z_{q}^{\langle-l\rangle}},
$$

where $a_{i}=\left\langle\mathrm{d} x_{i}^{\langle 1\rangle}, \Xi\right\rangle^{\langle-1\rangle}, b_{j k}=\left\langle\mathrm{d} u_{j}^{\langle k+1\rangle}, \Xi\right\rangle^{\langle-1\rangle}, c_{q l}=\left\langle\mathrm{d} z_{q}^{\langle-l+1\rangle}, \Xi\right\rangle^{\langle-1\rangle}, l \geq 0$. In the study below we stay in the space $\mathbf{X}$, therefore we will operate with the projections of $\Xi \in \mathcal{E}^{*}$ onto $\mathbf{X}$, denoted by $\Xi^{\pi}$ and defined as

$$
\Xi^{\pi}:=\sum_{i=1}^{n} \alpha_{i} \frac{\partial}{\partial x_{i}}
$$

Note, that the backward shift does not commute with the projection operator $\pi$.

Definition 2.1. (Albertini and Sontag, 1993) The reachable set from the state $x \in \bar{X}$ is the set of states $\mathcal{R}(x)$ to which one can move from $x$ via a finite number of forward shifts using some sequence of inputs. The controllable set to $x$ is the set of states $\mathcal{C}(x)$ from which one can move to $x$ via a finite number of forward shifts using some sequence of inputs. The reachable (controllable) set in $k$ steps from (to) $x$ is the set of states $\mathcal{R}_{k}(x)\left(\mathcal{C}_{k}(x)\right.$ ), to which (from which) one can move from $x$ (to $\left.x\right)$ in $k$ steps.

Definition 2.2. (Albertini and Sontag, 1993) The system (1) is called weakly reachable from $x \in \bar{X}$ (weakly controllable to $x \in \bar{X}$ ) if the interior of $\mathcal{R}(x)(\mathcal{C}(x)$ ) is a nonempty set. The system is called weakly reachable (controllable) in $k$ steps from (to) $x \in \bar{X}$, if $\mathcal{R}_{k}(x)\left(\mathcal{C}_{k}(x)\right)$ has a nonempty interior.

Definition 2.3. (Aranda-Bricaire, Kotta and Moog, 1996) A non-constant function $\phi(x)$ is called an autonomous variable of system (1), if there exists an integer $k \geq 1$ and a non-constant meromorphic function $\psi$ such that $\psi\left(\phi, \phi^{\langle 1\rangle}, \ldots, \phi^{\langle k\rangle}\right) \equiv 0$, where $\partial \psi / \partial \phi^{\langle k\rangle} \not \equiv 0$. System (1) is said to be generically accessible, if it has no autonomous variables.

Remark 1. It has been shown in (Albertini and Sontag, 1993), that the system (1) 
is generically accessible, if there is an open and dense subset $Y \subset \bar{X}$ such that for all $x \in Y$ the reachable set $\mathcal{R}(x)$ has a nonempty interior.

Recall the vector space $\mathcal{D}_{\infty}^{\pi} \subset \mathbf{X}$ (Mullari, Kotta, Bartosiewicz, Pawluszewicz and Moog, 2017):

$$
\mathcal{D}_{\infty}^{\pi}=\operatorname{span}_{\mathcal{K}}\left\{\left(\frac{\partial}{\partial u}\right)^{\langle-k\rangle \pi}, k>0\right\}
$$

is the union of

$$
\mathcal{D}_{k}^{\pi}=\operatorname{span}_{\mathcal{K}}\left\{\left(\frac{\partial}{\partial u}\right)^{\langle-l\rangle \pi}, l=1, \ldots, k\right\}, \quad k \geq 1 .
$$

There exists an index $k^{\star}$ such that $\operatorname{dim}_{\mathcal{K}} \mathcal{D}_{k-1}^{\pi}<\operatorname{dim}_{\mathcal{K}} \mathcal{D}_{k}^{\pi}$ for $k \leq k^{\star}$, but $\operatorname{dim}_{\mathcal{K}} \mathcal{D}_{k^{\star}}^{\pi}=$ $\operatorname{dim}_{\mathcal{K}} \mathcal{D}_{k^{\star}+1}^{\pi}=: \operatorname{dim}_{\mathcal{K}} \mathcal{D}_{\infty}^{\pi}$. According to its definition, $\mathcal{D}_{\infty}^{\pi}$ is invariant under the backward shift with projection, i.e. for each $\Xi \in \mathcal{D}_{\infty}^{\pi}$

$$
\Xi^{\langle-1\rangle \pi} \in \mathcal{D}_{\infty}^{\pi}
$$

Moreover, as shown in (Mullari, Kotta, Bartosiewicz, Pawluszewicz and Moog, 2017), for non-accessible system, each function $\phi(x)$, which is the invariant of $\mathcal{D}_{\infty}^{\pi}$, i.e. satisfies the condition $\langle\mathrm{d} \phi, \Xi\rangle \equiv 0$ for all $\Xi \in \mathcal{D}_{\infty}^{\pi}$, is also an autonomous variable and vice versa.

Theorem 2.4. (Mullari, Kotta, Bartosiewicz, Pawluszewicz and Moog, 2017) System (1) is generically accessible, iff

$$
\operatorname{dim}_{\mathcal{K}} \mathcal{D}_{\infty}^{\pi}=n
$$

Definition 2.5. The point $x \in \bar{X}$ is called a reachability (controllability) singular point of system (1), if the interior of $\mathcal{R}(x)$ (of $\mathcal{C}(x)$ ) is empty. It is called a singular point of reachability (controllability) in $k$ steps of system (1), if the interior of $\mathcal{R}_{k}(x)$ (of $\mathcal{C}_{k}(x)$ ) is empty.

\section{Reachability singular points}

The goal of this section is to show that the vector space $\mathcal{D}_{k}^{\pi}$ is helpful for finding the singular points, from which the system is not reachable in $k$ steps. Compute the $k$ th order forward shift of the states $x^{\langle k\rangle}=\bar{\Phi}^{k}\left(x, u, \ldots, u^{\langle k-1\rangle}\right)$, where

$$
\bar{\Phi}^{1}(x, u)=\bar{\Phi}(x, u), \bar{\Phi}^{k}\left(x, u, \ldots, u^{\langle k-1\rangle}\right)=\bar{\Phi}^{k-1}\left(\bar{\Phi}(x, u), u^{\langle 1\rangle}, \ldots, u^{\langle k-1\rangle}\right) .
$$

Compute $A:=\partial \bar{\Phi} / \partial x, B:=\partial \bar{\Phi} / \partial u$. Observe that the elements of the matrices $A$ and $B$ belong to $\mathcal{K}$. Denote

$$
x^{\langle k\rangle}=\bar{\Phi}^{k}\left(x, \mathbf{u}_{k}\right),
$$


where $\mathbf{u}_{k}:=\left(u, \ldots, u^{\langle k-1\rangle}\right)$. For a fixed initial state $x$, the map $\mathbf{u}_{k} \mapsto \bar{\Phi}^{k}\left(x, \mathbf{u}_{k}\right)$ assigns the state at time $k$ to a control sequence $\mathbf{u}_{k}$. Define the matrix

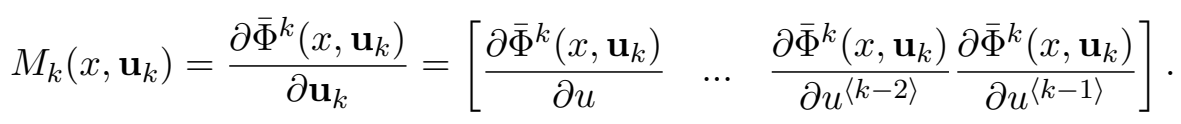

Proposition 3.1. The columns of matrix $M_{k}$ have the form:

$$
\frac{\partial \bar{\Phi}^{k}}{\partial u^{\langle k-1\rangle}}=B^{\langle k-1\rangle}, \quad \frac{\partial \bar{\Phi}^{k}}{\partial u^{\langle k-q\rangle}}=A^{\langle k-1\rangle} \ldots A^{\langle k-q+1\rangle} B^{\langle k-q\rangle}, q=2, \ldots, k .
$$

Proof. The proof is direct, taking into account (8) and using the chain rule.

Lemma 3.2. (Jakubczyk, Sontag, 1990) Let $x$ be fixed. Under Assumption 1, the set $\mathcal{R}_{k}(x)$ has a nonempty interior if and only if $\sup _{\mathbf{u}_{k} \in \mathbf{U}^{k}}\left\{\operatorname{rank}_{\mathbb{R}} M_{k}\left(x, \mathbf{u}_{k}\right)\right\}=n$, where $\mathbf{U}^{k} \subset \mathbb{R}^{k \times m}$. Consequently, the interior of $\mathcal{R}(x)$ is nonempty if and only if $\sup _{k>0}\left\{\sup _{\mathbf{u}_{k} \in \mathbf{U}^{k}} \operatorname{rank}_{\mathbb{R}} M_{k}\left(x, \mathbf{u}_{k}\right)\right\}=n$.

Consequently, for the values of $x$, where $\sup _{\mathbf{u}_{k} \in \mathbf{U}^{k}}\left\{\operatorname{rank}_{\mathbb{R}} M_{k}\left(x, \mathbf{u}_{k}\right)\right\}=n$, the system of equations (9) is locally solvable with respect to $\mathbf{u}_{k}$ and one can find a set of inputs necessary to take the system from a fixed $x$ into a desired final state $x^{\langle k\rangle} \in \bar{X}$. Lemma 3.2 allows to find the reachability singular points in $k$ steps and the reachability singular points. These points are found at locations where

$$
\sup _{\mathbf{u}_{k} \in \mathbf{U}^{k}}\left\{\operatorname{rank}_{\mathbb{R}} M_{k}\left(x, \mathbf{u}_{k}\right)\right\}<n,
$$

and

$$
\sup _{k>0}\left\{\sup _{\mathbf{u}_{k} \in \mathbf{U}^{k}}\left\{\operatorname{rank}_{\mathbb{R}} M_{k}\left(x, \mathbf{u}_{k}\right)\right\}\right\}<n,
$$

respectively.

The computation of the singular points from which the generically accessible system (1) is not weakly reachable, is addressed next. The points from which the system is not weakly reachable in $\mathrm{k}$ steps are characterized thanks to $D_{k}^{\pi}$. Compare the structures of the matrix $M_{k}$ and the matrix $N_{k}$, formed from the basis vector fields of $\mathcal{D}_{k}^{\pi}$ :

$$
N_{k}=\left[\left(\frac{\partial}{\partial u}\right)^{\langle-k\rangle \pi} \quad \cdots\left(\frac{\partial}{\partial u}\right)^{\langle-2\rangle \pi}\left(\frac{\partial}{\partial u}\right)^{\langle-1\rangle \pi}\right] .
$$

The forward and backward shifts of matrices are defined elementwise.

For an arbitrary vector field $\Xi=\alpha \partial / \partial x+\beta_{0} \partial / \partial u$ the coefficients $a$ of $\Xi^{\langle-1\rangle \pi}$ are computed according to (5): $\Xi^{\langle-1\rangle \pi}=\left\langle\mathrm{d} x^{\langle 1\rangle}, \Xi\right\rangle^{\langle-1\rangle} \partial / \partial x=$ $\langle\mathrm{d} \bar{\Phi}, \Xi\rangle^{\langle-1\rangle} \partial / \partial x$. Since $\bar{\Phi}$ depends only on $x$ and $u$, we get

$$
\Xi^{\langle-1\rangle \pi}=\left[A \alpha+B \beta_{0}\right]^{\langle-1\rangle} \frac{\partial}{\partial x} .
$$


Proposition 3.3. For any $q \geq 2$ holds the following:

$$
\left(\frac{\partial}{\partial u}\right)^{\langle-1\rangle \pi}=B^{\langle-1\rangle} \frac{\partial}{\partial x}, \quad\left(\frac{\partial}{\partial u}\right)^{\langle-q\rangle \pi}=A^{\langle-1\rangle} \ldots A^{\langle-q+1\rangle} B^{\langle-q\rangle} \frac{\partial}{\partial x} .
$$

Proof. Start with $\Xi=\partial / \partial u$ and compute the projection $\pi$ of its first order backward shift, using formula (13) and taking $\alpha \equiv 0$ and $\beta_{0}$ the $m \times m$ identity matrix, yielding directly (14). To compute the projections of the 2 nd order backward shift, apply the formula (13) now on the vector field (14), taking $\alpha=B^{\langle-1\rangle}$ and $\beta_{0} \equiv 0$ :

$$
\left(\frac{\partial}{\partial u}\right)^{\langle-2\rangle \pi}=\left(A B^{\langle-1\rangle}\right)^{\langle-1\rangle} \frac{\partial}{\partial x}=A^{\langle-1\rangle} B^{\langle-2\rangle} \frac{\partial}{\partial x}
$$

which proves (14) for $q=2$. Applying recursively formula (13) proves (14) for $q>$ 2 .

Corollary 3.4. If $M_{k}$ is the matrix defined by (10), then

$$
M_{k}=\sigma_{\Phi}^{k}\left(N_{k}\right) \text {. }
$$

Proof. Follows directly from Propositions 3.1, 3.3, and definition (12) of $N_{k}$.

The singular points for reachability in $k$ steps are characterized by the following theorem.

Theorem 3.5. Let $x$ be fixed. Under Asumption 1, the system is not weakly reachable from $x$ in $k$ steps if and only if

$$
\sup _{\mathbf{u}_{k} \in \mathbf{U}^{k}}\left\{\operatorname{rank}_{\mathbb{R}}\left[\sigma_{\Phi}^{k}\left(N_{k}\right)\right]\right\}<n .
$$

Proof. Follows directly from (11) and Corollary 3.4.

Example 3.6. Consider the system $x_{1}^{\langle 1\rangle}=\left(1+x_{1} u\right) \ln \left|x_{2}\right|, \quad x_{2}^{\langle 1\rangle}=e^{x_{1} u}$, defined for $x_{2}>0$. One can pick $z=u$, then the corresponding backward shift equations read

$$
x_{1}^{\langle-1\rangle}=\frac{\ln \left|x_{2}\right|}{z^{\langle-1\rangle}}, \quad x_{2}^{\langle-1\rangle}=\exp \left(\frac{x_{1}}{1+\ln \left|x_{2}\right|}\right) .
$$

The total differentials of the coordinate forward shifts are

$$
\begin{array}{rr}
\mathrm{d} x_{1}^{\langle 1\rangle}= & u \ln \left|x_{2}\right| \mathrm{d} x_{1}+\frac{1+x_{1} u}{x_{2}} \mathrm{~d} x_{2}+x_{1} \ln \left|x_{2}\right| \mathrm{d} u, \\
\mathrm{~d} x_{2}^{\langle 1\rangle}= & u e^{x_{1} u} \mathrm{~d} x_{1}+x_{1} e^{x_{1} u} \mathrm{~d} u .
\end{array}
$$

In order to determine the reachability singular points of this system, find first the points, from which the system is not weakly reachable in 2 steps and then examine the behaviour of these points under the forward shifts. Compute for this purpose the basis vector fields of $\mathcal{D}_{2}^{\pi}$. According to (5) and (6), we obtain the projection of the first order backward shift of $\partial / \partial u$ as the vector field $(\partial / \partial u)^{\langle-1\rangle \pi}=\left\langle\mathrm{d} x^{\langle 1\rangle}, \partial / \partial u\right\rangle^{\langle-1\rangle} \partial / \partial x$. Taking 
into account (19) yields $(\partial / \partial u)^{\langle-1\rangle \pi}=\left(x_{1} \ln \left|x_{2}\right|\right)^{\langle-1\rangle} \partial / \partial x_{1}+\left(x_{1} \exp \left(x_{1} u\right)\right)^{\langle-1\rangle} \partial / \partial x_{2}$. Due to (18) we get

$$
\left(\frac{\partial}{\partial u}\right)^{\langle-1\rangle \pi}=\frac{x_{1} \ln \left|x_{2}\right|}{u^{\langle-1\rangle}\left(1+\ln \left|x_{2}\right|\right)} \frac{\partial}{\partial x_{1}}+\frac{x_{2} \ln \left|x_{2}\right|}{u^{\langle-1\rangle}} \frac{\partial}{\partial x_{2}},
$$

The projection of the second order backward shift of $\partial / \partial u$ can be found analogously:

$$
\left(\frac{\partial}{\partial u}\right)^{\langle-2\rangle \pi}=\frac{\left.x_{1}\left[\left(1+\ln \left|x_{2}\right|\right)^{2}+\left(1+2 \ln \left|x_{2}\right|\right) x_{1}\right)\right]}{u^{\langle-2\rangle}\left(1+\ln \left|x_{2}\right|\right)\left(1+x_{1}+\ln \left|x_{2}\right|\right)} \frac{\partial}{\partial x_{1}}+\frac{x_{1} x_{2} \ln \left|x_{2}\right|}{u^{\langle-2\rangle}\left(1+x_{1}+\ln \left|x_{2}\right|\right)} \frac{\partial}{\partial x_{2}} .
$$

Writing now the matrix $N_{2}$ according to (12) and computing its second order forward shifts, we obtain due to (16) the matrix

$$
M_{2}=\left(\begin{array}{cc}
x_{1}\left[1+\left(2 u x_{1}+1\right) u^{\langle 1\rangle} \ln \left|x_{2}\right|\right] & u x_{1}\left(1+x_{1} u\right) \ln \left|x_{2}\right| \\
u^{\langle 1\rangle} x_{1} \ln \left|x_{2}\right| x_{2}^{\left(1+x_{1} u\right) u^{\langle 1\rangle}} & \left(1+x_{1} u\right) \ln \left|x_{2}\right| x_{2}^{\left(1+x_{1} u\right) u^{\langle 1\rangle}}
\end{array}\right) .
$$

Its generic rank is 2 , but $\sup _{\mathbf{u}_{2} \in \mathbf{U}^{2}}\left\{\operatorname{rank}_{\mathbb{R}}\left(M_{2}\right)\right\}$ is obviously less than 2 (i.e. the condition (17) is satisfied), if at least one of the following conditions holds:

$$
x_{1}=0, \quad x_{2}=1 .
$$

The constraints (22) give the states, from which the system is not reachable in 2 steps. If the first condition holds and the initial state is $\left(0, x_{2}\right)$, then for the next four time instances we get: $\left(\ln \left|x_{2}\right|, 1\right),\left(0, x_{2}^{u^{\langle 1\rangle}}\right),\left(u^{\langle 1\rangle} \ln \left|x_{2}\right|, 1\right),\left(0, x_{2}^{u^{\langle 1\rangle}} u^{\langle 3\rangle}\right)$. Consequently, the states of the system start to jump between the subspaces $x_{1}=0$ and $x_{2}=1$ regardless of the choice of inputs. The system behaves similarly, if one starts with the initial state $\left(x_{1}, 1\right)$. This means, the system is not weakly reachable from initial states $\left(1, x_{2}\right)$ and/or $\left(x_{1}, 1\right)$ also in more than 2 steps. Ergo, the system is not weakly reachable from any point in those subspaces of $\bar{X}$, defined by $x_{1}=0$ and/or $x_{2}=1$.

\section{Matrix $M_{k}$ does not define autonomous variables}

In this section it is shown that $M_{k}$ is of no help in finding directly the autonomous variables of the non-accessible systems, but for this purpose the vector space $\mathcal{D}_{\infty}^{\pi}$ is required.

Example 4.1. Consider the system

$$
x_{1}^{\langle 1\rangle}=u, \quad x_{2}^{\langle 1\rangle}=\ln \left|\frac{\exp \left(x_{2}\right) u}{x_{1}}\right|, \quad z=x_{1},
$$

resulting in the backward shifts

$$
x_{1}^{\langle-1\rangle}=z^{\langle-1\rangle}, \quad x_{2}^{\langle-1\rangle}=\ln \left|\frac{\exp \left(x_{2}\right) z^{\langle-1\rangle}}{x_{1}}\right|, \quad u^{\langle-1\rangle}=x_{1} .
$$


Compute, according to (13),

$$
\left(\frac{\partial}{\partial u}\right)^{\langle-1\rangle \pi}=\frac{\partial}{\partial x_{1}}+\frac{1}{x_{1}} \frac{\partial}{\partial x_{2}}, \quad\left(\frac{\partial}{\partial u}\right)^{\langle-2\rangle \pi}=0 .
$$

Obviously $\operatorname{dim}_{\mathcal{K}} \mathcal{D}_{\infty}^{\pi}=1<n=2$. According to Theorem 2.4 the system is not generically accessible. The space $\mathcal{D}_{\infty}^{\pi}$ has a single functionally independent invariant

$$
\phi(x)=x_{1} \exp \left(-x_{2}\right)
$$

which is the autonomous variable of system (23).

Compute the matrix $M_{2}$, using Proposition 3.1,

$$
M_{2}=\left(\begin{array}{cc}
0 & 1 \\
0 & 1 / u^{\langle 1\rangle}
\end{array}\right)
$$

Though the matrix $M_{2}$ has also generic rank 1, it is of no help to find the autonomous variable. Its left kernel

$$
\left[\begin{array}{ll}
-1 & u^{\langle 1\rangle}
\end{array}\right]
$$

as the row matrix is not related to $\phi(x)$ directly. However, expressing the gradient of $\phi$ as the row vector, we get $\left[\exp \left(-x_{2}\right),-x_{1} \exp \left(-x_{2}\right)\right]$. Shifting it twice forward elementwise, one gets the row vector

$$
\left[\frac{x_{1} \exp \left(-x_{2}\right)}{u^{\langle 1\rangle}},-x_{1} \exp \left(-x_{2}\right)\right]
$$

which is obtained via multiplying (24) by $-x_{1} \exp \left(-x_{2}\right) / u^{\langle 1\rangle}$, and therefore, belongs also to the left kernel of $M_{2}$. In plain words, the left kernel of $M_{2}$ does not define the autonomous variable itself but defines its 2-step forward shift.

To conclude, there are two related objects, allowing to check the accessibility of the system (1) - the matrix $M_{k}$, given by (10), and the vector space $\mathcal{D}_{k}^{\pi}$, given by (7). The first one allows additionally to find the singular points of weak reachability in $k$ steps, but does not reveal directly the autonomous variables in the straightforward manner. The second object enables to compute the autonomous variables directly, but not the reachability singular points. The matrix $M_{k}$ is obtained, shifting $k$ times forward the elements of the matrix (12), built from the basis vector fields of $\mathcal{D}_{k}^{\pi}$ as the column vectors.

\section{Controllability singular points}

In this section we shall consider the subclass of systems (1), satisfying the following assumption.

Assumption 2. Let $\bar{\Phi}_{u}(x)=\bar{\Phi}(x, u)$ and assume that for every $u$ the map $\bar{\Phi}_{u}$ has a global analytic inverse $\bar{\Phi}_{u}^{-1}$. Define $\Psi\left(x^{\langle 1\rangle}, u\right):=\bar{\Phi}_{u}^{-1}\left(x^{\langle 1\rangle}\right)$. Then the backward shift 
of the state is defined as

$$
x^{\langle-1\rangle}=\Psi\left(x, u^{\langle-1\rangle}\right) .
$$

Under the Assumption 2 the natural choice is $z=u$. Compute the $k$ th order backward shift of the state

$$
x^{\langle-k\rangle}=\Psi^{k}\left(x, u^{\langle-1\rangle}, \ldots, u^{\langle-k\rangle}\right)
$$

where $\quad \Psi^{1}\left(x, u^{\langle-1\rangle}\right) \quad:=\quad \Psi\left(x, u^{\langle-1\rangle}\right), \quad \Psi^{k}\left(x, u^{\langle-1\rangle}, u^{\langle-2\rangle}, \ldots, u^{\langle-k\rangle}\right) \quad=$ $\left(\Psi^{k-1}\left(x, u^{\langle-1\rangle}, u^{\langle-2\rangle}, \ldots, u^{\langle-k+1\rangle}\right)\right)^{\langle-1\rangle}=\Psi^{k-1}\left(\Psi\left(x, u^{\langle-1\rangle}\right), u^{\langle-2\rangle}, \ldots, u^{\langle-k\rangle}\right)$. Define the matrix

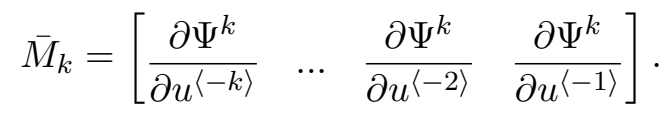

Lemma 5.1. Let $x$ be fixed. Under the Assumption 2, the set $\mathcal{C}_{k}(x)$ has a nonempty interior if and only if $\sup _{\overline{\mathbf{u}_{k}} \in \mathbf{U}^{k}}\left\{\operatorname{rank}_{\mathbb{R}} \bar{M}_{k}\left(x, \overline{\mathbf{u}}_{k}\right)\right\}=n$. Consequently, the interior of $\mathcal{C}(x)$ is nonempty if and only if $\sup _{k>0}\left\{\sup _{\overline{\mathbf{u}}_{k} \in \mathbf{U}^{k}} \operatorname{rank}_{\mathbb{R}} \bar{M}_{k}\left(x, \overline{\mathbf{u}}_{k}\right)\right\}=n$, where $\overline{\mathbf{u}}_{k}=$ $\left\{u^{\langle-k\rangle}, \ldots, u^{\langle-1\rangle}\right\}$.

Proof. The proof is analogous to the proof of Lemma 3.2.

Lemma 5.1 allows to find the controllability singular points in $k$ steps and the controllability singular points. These points are found at locations where

$$
\sup _{\overline{\mathbf{u}_{k} \in \mathbf{U}^{k}}}\left\{\operatorname{rank}_{\mathbb{R}} \bar{M}_{k}\left(x, \overline{\mathbf{u}}_{k}\right)\right\}<n,
$$

and

$$
\sup _{k>0}\left\{\sup _{\mathbf{u}_{k} \in \mathbf{U}^{k}}\left\{\operatorname{rank}_{\mathbb{R}} \bar{M}_{k}\left(x, \overline{\mathbf{u}}_{k}\right)\right\}\right\}<n,
$$

respectively.

The computation of the singular points to which the generically accessible system (1) is not weakly controllable in $k$ steps, is addressed next. Under Assumption 2 the matrix $A(x, u)$ is invertible for each $(x, u)$. Denote the inverse of $A$ by $\bar{A}$.

Proposition 5.2. Under Assumption 2, the elements of the matrix $\bar{M}_{k}$ in (25) have the form:

$$
\frac{\partial \Psi^{k}}{\partial u^{\langle-l\rangle}}=-\bar{A}^{\langle-k\rangle} \ldots \bar{A}^{\langle-l\rangle} B^{\langle-l\rangle}, \quad l=1, \ldots, k
$$

Proof. Taking the total differential of (1) yields $\mathrm{d} x^{\langle 1\rangle}=A \mathrm{~d} x+B \mathrm{~d} u$. Multiplying it by $\bar{A}$ from left and shifting the result one step backward gives $\mathrm{d} x^{\langle-1\rangle}=\bar{A}^{\langle-1\rangle} \mathrm{d} x-$ $\bar{A}^{\langle-1\rangle} B^{\langle-1\rangle} \mathrm{d} u^{\langle-1\rangle}$. Then direct computation gives

$$
\begin{aligned}
\mathrm{d} x^{\langle-k\rangle}= & \mathrm{d} \Psi^{k}=\bar{A}^{\langle-k\rangle} \ldots \bar{A}^{\langle-1\rangle} \mathrm{d} x-\bar{A}^{\langle-k\rangle} \ldots \bar{A}^{\langle-1\rangle} \bar{B}^{\langle-1\rangle} \mathrm{d} u^{\langle-1\rangle}- \\
& -\bar{A}^{\langle-k\rangle} \ldots \bar{A}^{\langle-2\rangle} \bar{B}^{\langle-2\rangle} \mathrm{d} u^{\langle-2\rangle}-\ldots-\bar{A}^{\langle-k\rangle} \bar{B}^{\langle-k\rangle} \mathrm{d} u^{\langle-k\rangle},
\end{aligned}
$$


From (28) follows directly (27).

Lemma 5.3. $\bar{M}_{k}=-\bar{A}^{\langle-k\rangle} \ldots \bar{A}^{\langle-1\rangle} N_{k}$.

Proof. Due to (27),

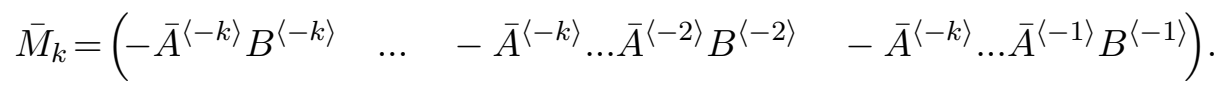

On the other hand, according to (12) and Proposition 3.3

$$
N_{k}=\left(\begin{array}{llll}
A^{\langle-1\rangle} \ldots A^{\langle-k-1\rangle} B^{\langle-k\rangle} & \ldots & A^{\langle-1\rangle} B^{\langle-2\rangle} & B^{\langle-1\rangle}
\end{array}\right) .
$$

Multiplying $N_{k}$ from the left hand side by $-\bar{A}^{\langle-k\rangle} \ldots \bar{A}^{\langle-1\rangle}$ and taking into account (29) proves the lemma.

Lemma 5.4. $\sup _{\overline{\bar{u}_{k} \in \mathbf{U}^{k}}}\left\{\operatorname{rank}_{\mathbb{R}} N_{k}\left(x, \overline{\mathbf{u}}_{k}\right)\right\}<n \Leftrightarrow \sup _{\overline{\mathbf{u}_{k} \in \mathbf{U}^{k}}}\left\{\operatorname{rank}_{\mathbb{R}} \bar{M}_{k}\left(x, \overline{\mathbf{u}_{k}}\right)\right\}<n$.

Proof. According to Sylvester inequality, $\operatorname{rank}_{\mathbb{R}}(P Q) \leq \min \left\{\operatorname{rank}_{\mathbb{R}} P, \operatorname{rank}_{\mathbb{R}} Q\right\}$. Due to Lemma 5.3, $\operatorname{rank}_{\mathbb{R}} \bar{M}_{k} \leq \operatorname{rank}_{\mathbb{R}} N_{k}$, and so $\operatorname{rank}_{\mathbb{R}} N_{k}<n$ yields $\operatorname{rank}_{\mathbb{R}} \bar{M}_{k}<n$. Since, again by Lemma $5.3, N_{k}=-A^{\langle-1\rangle} \ldots A^{\langle-k\rangle} \bar{M}_{k}$, and so $\operatorname{rank}_{\mathbb{R}} N_{k} \leq$ $\operatorname{rank}_{\mathbb{R}} \bar{M}_{k}$, proving the lemma.

Theorem 5.5. Under Assumption 2, the system (1) is not weakly controllable to a fixed point $x \in \bar{X}$ in $k$ steps if and only if

$$
\sup _{\overline{\mathbf{u}}_{k} \in \overline{\mathbf{U}}^{k}} \operatorname{rank}_{\mathbb{R}} N_{k}\left(x, \overline{\mathbf{u}_{k}}\right)<n,
$$

or equivalently

$$
\sup _{\overline{\mathbf{u}_{k}} \in \overline{\mathbf{U}}^{k}} \operatorname{dim}_{\mathbb{R}} \mathcal{D}_{k}^{\pi}\left(x, \overline{\mathbf{u}_{k}}\right)<n .
$$

Proof. Follows directly from (26) and Lemma 5.4.

Example 5.6. (Continuation of Example 3.6). The system satisfies Assumption 2, which allowed us to take $z=u$. Find the controllability singular points in 2 steps according to Theorem 5.5, using the vector space $\mathcal{D}_{2}^{\pi}$. The generic dimension of $\mathcal{D}_{2}^{\pi}$ is 2 , i.e. the system is generically accessible, but the dimension of $\sup _{\overline{\mathbf{u}}_{2} \in \mathbf{U}^{2}} \operatorname{dim}_{\mathbb{R}} \mathcal{D}_{2}^{\pi}\left(x, \overline{\mathbf{u}}_{2}\right)$ drops, if at least one of the following conditions holds:

$$
x_{1}=0, \quad x_{2}=1,
$$

Moreover, the basis vector fields of $\mathcal{D}_{2}^{\pi}$ are not defined at the following subspaces of $\bar{X}$ :

$$
1+\ln \left|x_{2}\right|=0 \Rightarrow x_{2}=e^{-1}, \quad 1+x_{1}+\ln \left|x_{2}\right|=0 \Rightarrow x_{2}=e^{-1-x_{1}}
$$


Check whether the states determined from the conditions (30) and (31) are really the controllability singular points. For this purpose compute, using (18),

$$
x_{1}^{\langle-2\rangle}=\frac{x_{1}}{u^{\langle-2\rangle}\left(1+\ln \left|x_{2}\right|\right)}, \quad x_{2}^{\langle-2\rangle}=\exp \left(\frac{\ln \left|x_{2}\right|\left(1+\ln \left|x_{2}\right|\right)}{u^{\langle-1\rangle}\left(1+x_{1}+\ln \left|x_{2}\right|\right)}\right),
$$

where $z^{\langle-1\rangle}$ is replaced by $u^{\langle-1\rangle}$. Examine (18) and (32). If the first condition $x_{1}=0$ in (30) holds, then $(18)$ gives $x_{2}^{\langle-1\rangle}=1$, and (32) yields $x_{1}^{\langle-2\rangle}=0$, i.e. $\mathcal{C}_{2}(x)$ for the subspace $x_{1}=0$ are the one-dimensional spaces $x_{1}=0$ and $x_{2}=1$, which have an empty interior and therefore the subspace $x_{1}=0$ is really weakly non-controllable. Analogously can be shown, that the subspace $x_{2}=1$ is also weakly non-controllable.

Examine next the conditions (31). Show first, that the subspace defined by $x_{2}=$ $e^{-1}$ is not weakly controllable in two steps, i.e. one cannot move the system into an arbitrary point of this subspace in two steps. For this purpose write the second order forward shifts of the state coordinates

$$
x_{1}^{\langle 2\rangle}=\left[1+\left(1+x_{1} u\right) u^{\langle 1\rangle} \ln \left|x_{2}\right|\right] x_{1} u, \quad x_{2}^{\langle 2\rangle}=x_{2}^{\left(1+x_{1} u\right) u^{\langle 1\rangle}},
$$

and try to compute the values of inputs, necessary to move the system from an arbitrary initial state into the subspace $x_{2}=e^{-1}$. Writing the second formula of (33) in alternative form $x_{2}^{\langle 2\rangle}=\exp \left[\left(1+x_{1} u\right) \ln \left|x_{2}\right| u^{\langle 1\rangle}\right]$ and setting $x_{2}=e^{-1}$ yields $1+\left(1+x_{1} u\right) \ln \left|x_{2}\right| u^{\langle 1\rangle}=0$. Due to the first formula of (33) one obtains $x_{1}^{\langle 2\rangle}=0$ independent of the choice of inputs. Ergo, the final state $\left(x_{1}^{\langle 2\rangle}, e^{-1}\right)$ is not weakly controllable except the point $\left(0, e^{-1}\right)$. Analogously one can show, that the subspace $x_{2}=\exp \left(-1-x_{1}\right)$ is also not controllable, i.e. one can not move the system from an arbitrary initial state into the final state $\left(x_{1}^{\langle 2\rangle}, \exp \left(-1-x_{1}\right)\right)$ except the point $\left(1, \exp \left(-1-x_{1}\right)\right)$.

Example 5.7. As another, well-known example, consider a loop of wire, rotating around a vertical axis in an homogeneous magnetic field with the horizontal field lines. The system of equations, characterizing the rotational motion have, in case of a small rotational angles, the following form:

$$
x_{1}^{\langle 1\rangle}=x_{1}+x_{2} T, \quad x_{2}^{\langle 1\rangle}=x_{2}+\left(a x_{1} u-b x_{2}\right) T,
$$

where $x_{1}$ and $x_{2}$ are the rotational angle and the angular velocity of the loop, respectively, $T$ is the sampling time. The system constants are $a=B S / J, b=\mu / J$, where $B$ is the magnetic induction, $S$ - the area and $J$ - the moment of inertia of the loop, and $\mu$-the frictional coefficient. Taking $z=u$, we obtain the corresponding backward shift equations:

$$
x_{1}^{\langle-1\rangle}=\frac{1}{\Lambda_{1}}\left[\left(b x_{1}+x_{2}\right) T-x_{1}\right], \quad x_{2}^{\langle-1\rangle}=\frac{1}{\Lambda_{1}}\left[u^{\langle-1\rangle} x_{1} a T-x_{2}\right],
$$

where $\Lambda_{1}=u^{\langle-1\rangle} a T^{2}+b T-1$. Compute the projections of the backward shifts of 
$\partial / \partial u$ according to (5) and (6):

$$
\begin{aligned}
\left(\frac{\partial}{\partial u}\right)^{\langle-1\rangle \pi}= & \frac{a}{\Lambda_{1}}\left[\left(x_{1} b+x_{2}\right) T^{2}-x_{1} T\right] \frac{\partial}{\partial x_{2}} \\
\left(\frac{\partial}{\partial u}\right)^{\langle-2\rangle \pi}= & \frac{a}{\Lambda_{2}}\left(\left[x_{1} b^{2}+x_{2} b+u^{\langle-1\rangle} x_{1} a T^{4}-2\left(x_{1} b+x_{2}\right) T^{3}+x_{1} T^{2}\right] \frac{\partial}{\partial x_{1}}+\right. \\
+ & {\left[\left(x_{1} b^{3}+x_{2} b^{2}+u^{\langle-1\rangle} x_{1} a b\right) T^{4}-\left(3 x_{1} b^{2}+3 x_{2} b+u^{\langle-1\rangle} x_{1} a\right) T^{3}+\right.} \\
& \left.\left.+\left(3 x_{1} b+2 x_{2}\right) T^{2}-x_{1} T\right] \frac{\partial}{\partial x_{2}}\right),
\end{aligned}
$$

where $\Lambda_{2}=u^{\langle-2\rangle} u^{\langle-1\rangle} a^{2} T^{4}+\left(u^{\langle-1\rangle}+u^{\langle-1\rangle}\right) a b T^{3}+\left[b^{2}-\left(u^{\langle-1\rangle}+u^{\langle-2\rangle}\right) a\right] T^{2}-$ $2 b T+1$. The system is generically accessible, because $\operatorname{dim}_{\mathcal{K}} \mathcal{D}_{2}^{\pi}=2$, but $\sup _{\overline{\mathbf{u}}_{2} \in U^{2}} \operatorname{dim}_{\mathbb{R}} \mathcal{D}_{2}^{\pi}\left(x, \overline{\mathbf{u}}_{2}\right)$ drops, when at least one the following conditions holds:

$$
x_{1}=x_{2}=0, \quad x_{2}=x_{1}(1-b T) / T .
$$

In order to check the weak controllability in 2 steps of subspaces (35) we find the states, from which one can steer the system into these subspaces. Taking first the point $(0,0)$ one can find with the help of $(34)$, that the single possible previous state is again $(0,0)$, i.e. the point $(0,0)$ is not weakly controllable in any number of steps.

For the subspace $x_{2}=x_{1}(1-b T) / T$ we obtain, according to (34), that $x^{\langle-1\rangle}=\left(0, x_{1} / T\right)$, which is a single point. The next step backward gives $x^{\langle-2\rangle}=$ $\left(x_{1} / \Lambda_{1}^{\langle-1\rangle}, x_{1} /\left(\Lambda_{1}^{\langle-1\rangle} T\right)\right)$, which, depending on $u^{\langle-2\rangle}$, is a line. But the third backward shift yields $x^{\langle-3\rangle}=\left(x_{1}(b T-2) / \Lambda_{3}, x_{1}\left(1+a u^{\langle-3\rangle} T^{2}\right) / \Lambda_{3}\right)$, where $\Lambda_{3}=u^{\langle-2\rangle} u^{\langle-3\rangle} a^{2} T^{4}+$ $\left(u^{\langle-2\rangle}+u^{\langle-3\rangle}\right) a b T^{3}+\left[b^{2}-\left(u^{\langle-2\rangle}+u^{\langle-3\rangle}\right) a\right] T^{2}-2 b T+1$. Depending on the choice of $u^{\langle-2\rangle}$ and $u^{\langle-3\rangle}$, this covers the entire $\bar{X}$. Ergo, the subspace $x_{2}=x_{1}(1-b T) / T$ is weakly controllable in 3 steps.

\section{Conclusion}

The vector space, introduced in (Mullari, Kotta, Bartosiewicz, Pawluszewicz and Moog, 2017) as the discrete-time analogue of the strong accessibility distribution, unlike its continuous-time counterpart, does not directly identify the states from which the system is not weakly reachable in $k$ steps. In order to find such states, a new object is constructed as described below. Writing the vector space in the matrix form (i.e. taking its basis vector fields as the columns of the matrix), the elements of the matrix as scalar functions are shifted $\mathrm{k}$ times forward. This shifted matrix enables to find directly the points from which the generically accessible system is not weakly reachable in $\mathrm{k}$ steps. However, from the other side, the shifted matrix is of no help to find the autonomous variables of the system. To conclude, in the discrete-time case, unlike in the continuous-time case, two related objects are needed. Finally note that each of these two objects allows to check generic accessibility property. However, the controllability singular points in $k$ steps can be found using the analogue of the strong accessibility distributions. 


\section{References}

Albertini, F., Sontag, E.D. (1993). Discrete-time transitivity and accessibility: Analytic systems, SIAM Journal of Control and Optimization, 31(6), 1599-1622.

Aranda-Bricaire, E., Kotta, Ü., Moog, C.H. (1996). Linearization of discrete-time systems, SIAM Journal of Control and Optimization, 34(6), 1999-2023.

Conte, C., Moog, C.H., Perdon, A.M. (2007) Algebraic Methods for Nonlinear Control Systems, Springer-Verlag, London.

Dieudonné, J. (1960) Foundations of Modern Analysis, Academic Press Inc.

Hanba, S. (2017). Controllability to the origin implies state-feedback stabilizability for discretetime nonlinear systems, Automatica, 76 (2), 49-52.

Jakubczyk, B., Sontag, E.D. (1990). Controllability of nonlinear discrete-time systems: A Liealgebraic approach, SIAM Journal of Control and Optimization, 28(1), 1-33.

Kawano, Y., Ohtsuka, T. (2013). Simple sufficient conditions for reachability of discrete-time polynomial systems, IEEE Transactions on Automatic Control, 58(12), 3203-3206.

Kawano, Y., Ohtsuka, T. (2016). Commutative algebraic methods for controllability of discretetime polynomial systems, International Journal of Control, 89(2), 343-351.

Mullari, T., Kotta, Ü., Bartosiewicz, Z., Pawluszewicz, E. Moog, C.H.(2017) Forward and backward shifts of vector fields: towards the dual algebraic framework, IEEE Transactions on Automatic Control, 62(6), 3029-3033.

Rieger, K., Schlacher, K. (2011). Implicit discrete-time systems and accessibility, Automatica, $47(9), 1849-1859$. 